INPLASY

PROTOCOL

To cite: Yu et al. Effectiveness and safety of moxibustion for menopausal obesity: a protocol for systematic review and meta-analysis. Inplasy protocol 202220087. doi: 10.37766/inplasy2022.2.0087

Received: 20 February 2022

Published: 21 February 2022

Corresponding author:

Zhongyu Zhou

1070876798@qq.com

Author Affiliation:

Hubei University of Chinese

Medicine.

Support: 0686-204001171345N.

Review Stage at time of this submission: The review has not yet started.

Conflicts of interest:

None declared.

\section{Effectiveness and safety of moxibustion for menopausal obesity: a protocol for systematic review and meta-analysis}

Yu, B1; Wang, J2; Zhang, Y3; Zhang, Y4; Xu, K5; Huang, W6; Zhou, Z7.

Review question / Objective: The aim of this protocol is to summarize the evidence from clinic trails of moxibustion for the treatment of menopausal obesity and to evaluate the effectiveness and safety of this intervention.

Condition being studied: The Global Burden of Disease Obesity Collaborators estimated that a total of 603.7 million adults had obesity, and the global prevalence of overweight or obesity is $39 \%$ to $49 \%$. Obesity not only significantly reduces patients'quality of life, increases the risk of illness (eg, diabetes, hypertension, and cardiovascular disease) and mortality, but also leads to mental illness (eg, depression, unwarranted stigma, and schizophrenia) and raises health care costs in countries in all parts of the world. Menopause obesity may increase the risk of breast cancer, endometrial, colon cancer, type 2 diabetes, cardiovascular disease, and obesity was associated with a $25 \%$ to $30 \%$ relative increase in breast cancer specific and overall mortality in menopausal women.

INPLASY registration number: This protocol was registered with the International Platform of Registered Systematic Review and Meta-Analysis Protocols (INPLASY) on 21 February 2022 and was last updated on 21 February 2022 (registration number INPLASY202220087).

\section{INTRODUCTION}

Review question / Objective: The aim of this protocol is to summarize the evidence from clinic trails of moxibustion for the treatment of menopausal obesity and to evaluate the effectiveness and safety of this intervention.

Rationale: Obesity is a multi-factorial nature resulting from genetic, physiological, behavioural, socio-cultural, 
and environmental factors which leads to an imbalance between energy intake and expenditure during an extended time period. Menopause is a period when the level of FSH increases. Due to neuroendocrine dysfunction, increased glucocorticoid secretion, decreased metabolic rate and other reasons, women are prone to a sharp increase in visceral fat during this life stage. Moxibustion has been widely used to treat endocrine system diseases, but evidence for its effectiveness and safty remains scarce at present.

Condition being studied: The Global Burden of Disease Obesity Collaborators estimated that a total of 603.7 million adults had obesity, and the global prevalence of overweight or obesity is $39 \%$ to $49 \%$. Obesity not only significantly reduces patients'quality of life, increases the risk of illness (eg, diabetes, hypertension, and cardiovascular disease) and mortality, but also leads to mental illness (eg, depression, unwarranted stigma, and schizophrenia) and raises health care costs in countries in all parts of the world. Menopause obesity may increase the risk of breast cancer, endometrial, colon cancer, type 2 diabetes, cardiovascular disease, and obesity was associated with a $25 \%$ to $30 \%$ relative increase in breast cancer specific and overall mortality in menopausal women.

\section{METHODS}

Search strategy: Nine key electronic databases, including The Cochrane Library, PubMed, Medline, EMBASE, Chinese Biomedical Literature (CBM), Chongqing Chinese Science and Technology Periodical Database (VIP), China National Knowledge Infrastructure (CNKI), and Wanfang Database was searched since the establishment of the database. The search was limited to studies in Chinese and English and used the following searchterms:"obesity", "overweight", "menopause", "climacteric", "involutional", "perimenopause", "menopausal obesity", "moxibustion".
Participant or population: We included patients diagnosed with menopausalobesity.

Intervention: Interventions in the treatment group included any kind of moxibustion, such as warm needling moxibustion, moxa burner moxibustion, indirect moxibustion, moxa stick moxibustion, moxa cone moxibustion, suspended moxibustion, thunder-fire wonder moxibustion, etc.

Comparator: We included any study that includes comparators.

Study designs to be included: The review will include randomised controlled trials (RCTs) investigating the efficacy and safety of moxibustion for patients with menopausal obesity in Chinese and English. Crossover trials will be excluded.

Eligibility criteria: Studies that met the following criteria will be included: (1) RCTs; (2) Preventions were focused on moxibustion for the treatment of menopausal obesity; (3) Primary outcomes included waist circumference and the modified Kupperman score.Studies were excluded if:(1)Grey literature such as nonRCTs reviews, case reports, mechanism of disease, animal experimentals, expert experience and conference articles will be excluded.(2) Articles published with incomplete data, or complete articles could not be obtained even after contacting the authors.

Information sources: To identify original studies suitable for answering the research questions, we searched The Cochrane Library, PubMed, Medline, EMBASE, CBM, VIP, CNKI, and Wanfang databases since the establishment of the database. The International Prospective Register of Systematic Reviews (PROSPERO), the Chinese Clinical Trial Registry Center and Clinical Trials will also be searched for ongoing trials. The search was limited to studies in Chinese and English and used the following searchterms: "obesity", "overweight", "menopause", "climacteric", "involutional", "perimenopause", "menopausal obesity", "moxibustion". 
Main outcome(s): Waist circumference and the modified Kupperman score.

Additional outcome(s): The Impact of Weight on Quality of Life Questionnaire (IWQOL-Lite) scale; the MOS Item Short from Health Survey (SF-36) scale; the Traditional Chinese Medicine (TCM) clinical symptoms; Obesity-related indicators: Weight (WG), body mass index (BMI), hip circumference (HC), waist-hip-ratio (WHR) and body fat percentage (F\%); Lipid metabolism outcomes: Blood glucose and insulin parameters, total cholesterol (TC), triglycerides (TG), high-density lipoprotein (HDL) and low-density lipoprotein (LDL); Sex hormone levels: Estradiol (E2), follicle stimulating hormone (FSH), luteinising hormone (LH) and the incidences of adverse reactions.

Data management: Two independent reviewers (YZ and KX) will use Excel 2013 software to extract the following information from eligible studies according to the Cochrane Handbook 5.2.0 guidelines: 1.Study information: Country, first author, title, journal, publication date, random sequence generation, allocation concealment and blind method. 2. Participants characteristics: race, region, sample size, average age, duration of disease, symptoms and signs for menopausal obesity. 3. Interventions and comparators: Type, dosage forms, duration and frequency of interventions and comparators, acupoint selection of moxibustion. 4.Outcomes: Primary and secondary outcomes, duration of follow-up and adverse events. Discrepancies noticed in the process of data cross-checking will be resolved through discussion or by the third reviewer (BY).

Quality assessment / Risk of bias analysis: Two reviewers (JW and YZ) will independently assess the methodological quality for each RCTs based on the Cochrane Collaboration risk of bias tool, including seven domains: Random sequence generation, allocation concealment, blinding of participants and personnel, blinding of outcome data, incomplete outcome data, selective reporting and other bias35. The studies will be evaluated as being of'low risk of bias,' 'high risk of bias,'or'unclear risk of bias'. Any disagreement will be resolved by consensus or arbitrated by the third rater (BY).

Strategy of data synthesis: Data will be synthesised and analysed by RevMan V.5.3. Meta-analysis will be performed using a fixed effect model if the heterogeneity is not statistically significant $(p \geq 0.10$ and $\left.I^{2} \leq 50 \%\right)$. If there is statistical heterogeneity among the results, the source of heterogeneity will be further analysed and a random-effect model will be used to for meta-analysis. Besides, significant clinical heterogeneity will be processed by subgroup analysis or sensitivity analysis, or descriptive analysis only.

Subgroup analysis: Subgroup analysis will be performed based on the factors that may influence the effect of intervention. The following subgroups will be investigated respectively: different types of moxibustion (warm needling moxibustion, moxa burner moxibustion, indirect moxibustion, moxa stick moxibustion, moxa cone moxibustion, suspended moxibustion, thunder-fire wonder moxibustion, etc.), different treatment schemes in the control group (sham moxibustion, pharmacotherapy, blank control), and treatment duration.

Sensitivity analysis: Sensitivity analysis will be conducted by iteratively excluding single studies with small sample size or low evidence. If there is no significant change in the outcomes after elimination, it indicates the robustness and stability of the meta-analysis results.

Language: English and Chinese.

Country(ies) involved: China.

Keywords: Moxibustion, menopausal obesity, systematic review, meta-analysis, protocol. 
Dissemination plans: The findings will be published in peer-reviewed journals and/or presented at scientific conferences.

Contributions of each author:

Author 1 - Benlu Yu.

Author 2 - Jiajie Wang.

Author 3 - Yanji Zhang.

Author 4 - Yingrong Zhang.

Author 5 - Kou Xu.

Author 6 - Wei Huang.

Author 7 - Zhongyu Zhou. 